\title{
Variáveis psicomotoras, cognitivas e funcionais em idosas saudáveis e com doença de Alzheimer
}

\author{
Psychomotor, cognitive, and functional variables in healthy older women and older women \\ with Alzheimer's disease
}
Variables psicomotoras, cognitivas y funcionales en ancianas sanas y con enfermedad de Alzheimer Soraia Fernandes das Neves Glisoi', Thays Martins Vital da Silva², Ruth Ferreira Galduróz ${ }^{3}$

RESUMO I A teoria da retrogênese descreve os declínios apresentados na doença de Alzheimer a partir dos estágios do desenvolvimento neuropsicomotor de Piaget. O objetivo deste estudo transversal é avaliar e descrever aspectos psicomotores, cognitivos e quedas, investigando a relação de dependência entre essas variáveis de acordo com a teoria da retrogênese. A amostra foi composta por 45 idosas (27 saudáveis e 18 com Alzheimer fase leve), residentes em São Paulo entre 2016 e 2017. Para a avaliação foram utilizados: anamnese, Escala de Depressão Geriátrica de Yesavage (GDS-30), Montreal Cognitive Assessment (MoCA), Cambridge Cognitive Exam-Revised (CAMCOG-R), Escala de Equilíbrio de Berg (EEB), timed up and go test (TUGT), Avaliação Direta do Estado Funcional (DAFS-BR), teste $U$ de Mann-Whitney, coeficiente de correlação de Spearman e análise de regressão múltipla com $p<0,05$. Observou-se ordem de perda semelhante à sugerida pela teoria da retrogênese nos dois grupos estudados. Quanto menor o índice CAMCOG-R, maior o número de quedas $(p=0,03)$. Itens praxia $(p<0,00)$ e funções executivas $(p<0,00)$ do CAMCOG-R mostraramse altamente correlacionados com o TUGT e a EEB. Sugere-se relação de dependência entre funcionalidade e cognição e equilíbrio e cognição, bem como correlação entre risco de queda e desempenho cognitivo nos dois grupos estudados. As perdas observadas estão de acordo com o que a teoria da retrogênese propõe, mas com diferentes intensidades entre os grupos. Estudos longitudinais são necessários, com uso de exames de imagem para validar a teoria nos padrões de perda em idosos com e sem doença de Alzheimer.

Palavras-chave | Envelhecimento; Teoria da Retrogênese; Doença de Alzheimer.

ABSTRACT I The Retrogenesis Theory (RT) describes the declines presented in Alzheimer's disease (AD) based on the stages of Piaget's neuropsychomotor development. The objective of this cross-sectional study was to evaluate and describe psychomotor and cognitive aspects, falls and the dependency relations between these variables according to RT in healthy older women and with probable Alzheimer's Disease (AD). Composed of 45 older women (27 healthy and 18 with mild AD), residing in São Paulo from 2016 to 2017. Anamnesis, Geriatric Depression Scale Yesavage (GDS-30), Montreal Cognitive Assessment (MoCA), Cambridge were used for evaluation Revised Cognitive Exam (CAMCOG-R), Berg Balance Scale (BBS), timed up and go test (TUGT) and Direct Functional Status Assessment (DAFS-BR). The Mann-Whitney U tests, Spearman correlation and multiple regression analysis were used with $\mathrm{p}<0.05$. A loss order similar to that suggested by RT was observed in both groups. The lower the CAMCOG-R index, the greater the number of falls $(p=0.03)$. Praxia items $(p<0.00)$ and executive functions $(p<0.00)$ of CAMCOG-R highly correlated with TUGT and BBS. A dependency relation between functionality/cognition is suggested; balance/cognition and correlation between risk of falling and cognitive performance in both groups. The observed

Estudo realizado na Universidade Federal do ABC (UFABC), campus São Bernardo do Campo, na Fundação Nossa Senhora Auxiliadora do Ipiranga (Funsai) e Associação de Familiares e Amigos do Idoso (Afai), localizados em São Paulo.

Universidade Federal do ABC (UFABC) - São Bernardo do Campo (SP), Brasil. E-mail: soraia.glisoi.sg@gmail.com.

Orcid: 0000-0001-8607-9968

Instituto Federal Goiano - Morrinhos (GO), Brasil. E-mail: thaysmv87@gmail.com. Orcid: 0000-0002-9523-1156

3Universidade Federal do ABC (UFABC) - Santo André (SP), Brasil. E-mail: ruth.galduroz@ufabc.edu.br. Orcid: 0000-0002-0778-1087

Endereço para correspondência: Soraia Fernandes das Neves Glisoi - Rua Dona Angelina Santeo Chiozzani, 119, ap. 11, bloco 5 - Mauá (SP), Brasil - CEP: 09360-740 E-mail: soraia.glisoi.sg@gmail.com - Fonte de financiamento: nada a declarar - Conflito de interesses: nada a declarar - Apresentação: 26 maio 2020 - Aceito para publicação: 7 fev. 2021 - Aprovado pelo Comitê de Ética: Protocolo nº 54028316.6.0000.5594. 
losses are in line with what the RT proposes, but with different intensities between the groups. Longitudinal studies are necessary, with the use of imaging tests to validate RT in the loss patterns in older people with and without AD.

Keywords | Aging; Retrogenesis Theory; Alzheimer's Disease.

RESUMEN I La teoría de la retrogénesis describe el proceso de degeneración de la enfermedad de Alzheimer desde las etapas de desarrollo neuropsicomotor de Piaget. El objetivo de este estudio transversal es evaluar y describir los aspectos psicomotores, cognitivos y de caídas, y la relación de dependencia entre estas variables según la teoría de la retrogénesis. La muestra estuvo conformada por 45 ancianas (27 sanas y 18 con Alzheimer leve), residentes en São Paulo entre 2016 y 2017. En la evaluación se utilizaron: Anamnesis, Escala de Depresión Geriátrica de Yesavage (GDS-30), Evaluación Cognitiva de Montreal (MoCA), Cambridge Cognitive Exam-Revised (CAMCOG-R), Escala de Equilibrio de Berg (EEB), timed up and go test (TUGT), Evaluación Directa del Estado Funcional (DAFS-BR), prueba U de Mann-Whitney, coeficiente de correlación de Spearman y análisis de regresión múltiple con $p<0,05$. Se observó un orden de pérdida similar al sugerido por la teoría de la retrogénesis en los dos grupos estudiados. Cuanto menor es el índice CAMCOG-R, mayor el número de caídas $(p=0,03)$. Los ítems de praxia $(p<0,00)$ y las funciones ejecutivas $(p<0,00)$ de CAMCOG-R demostraron estar altamente correlacionados con TUGT y EEB. Se sugiere una relación de dependencia entre funcionalidad y cognición y equilibrio y cognición, así como una correlación entre el riesgo de caídas y el desempeño cognitivo en los dos grupos estudiados. Las pérdidas observadas están acorde con lo que propone la teoría de la retrogénesis, pero con diferentes intensidades entre los grupos. Son necesarios estudios longitudinales utilizando pruebas de imagen para validar la teoría en patrones de pérdida en personas mayores con y sin la enfermedad de Alzheimer. Palabras clave | Envejecimiento; Teoría de la Retrogénesis; Enfermedad de Alzheimer.

\section{INTRODUÇÃO}

Causa mais comum de demência, responsável por $50 \%$ a $75 \%$ de todos os casos ${ }^{1}$, a doença de Alzheimer (DA) é um processo neurodegenerativo, multifatorial, caracterizado pelo acúmulo de beta-amilóide em placas senis e fosforilação da proteína tau em emaranhados neurofibrilares. Esses fenômenos resultam em perdas sinápticas e neuronais que alteram as funções cognitivas e causam danos à memória, à atenção e ao raciocínio ${ }^{2,3}$. Além do comprometimento cognitivo, os idosos com DA apresentam alterações motoras e funcionais que impactam diretamente a qualidade de vida e a sobrevida ${ }^{4,5}$.

Primeira tentativa de relacionar a demência ao desenvolvimento humano, a teoria da retrogênese (TR) procura explicar os declínios apresentados na DA a partir dos estágios do desenvolvimento neuropsicomotor de Piaget ${ }^{6}$. De acordo com a teoria, as perdas cognitivas na DA ocorrem no reverso da ordem de aquisição neuropsicomotora proposta por Piaget, ou seja, nas fases iniciais as habilidades mais complexas se perdem e, consequentemente, as demandas cognitivas aumentam, enquanto nas etapas finais são necessárias habilidades mais primitivas e, portanto, há menos demandas cognitivas ${ }^{6,7}$.

Embora já bem definida, a TR descreve apenas declínios cognitivos e funcionais, que são isolados, sem avaliar a relação entre variáveis cognitivas e motoras ou cognitivas e funcionais. Também não estão claras a ordem das perdas motoras na DA e a influência da cognição nas variáveis funcionais e motoras nos estágios iniciais da doença, bem como no risco de quedas. As hipóteses do presente estudo são: (1) as primeiras perdas (cognitivas, motoras e funcionais) na DA seriam as de funções mais complexas, relacionadas à perda de funções cognitivas específicas (executivas e praxia); (2) as perdas ocorreriam em ordem inversa à aquisição neuropsicomotora proposta por Piaget; (3) haveria correlação e relação de dependência entre cognição e funcionalidade, cognição e aspectos motores. Para elucidar essas hipóteses, o estudo avalia e descreve aspectos psicomotores, cognitivos e funcionais, utilizando o modelo TR para investigar a relação de dependência entre essas variáveis em idosas saudáveis e em idosas diagnosticadas com DA provável na fase inicial.

\section{METODOLOGIA}

\section{População e desenho de estudo}

Estudo transversal, com amostra composta por mulheres de 60 a 90 anos, residentes em São Paulo e Região Metropolitana e incluídas em dois serviços 
de apoio ao idoso: centro de convivência "VivaVida", da Fundação Nossa Senhora Auxiliadora do Ipiranga (Funsai), e centro-dia Associação de Familiares e Amigos de Idosos (Afai).

Para participar do estudo, as idosas não deveriam apresentar alterações sensoriais descompensadas (comprometimento da visão grave sem o uso de óculos, diminuição da acuidade auditiva sem o uso de prótese ou alterações vestibulares não tratadas), alterações ortopédicas graves (osteoartrose grave, fratura com deformidade, deformidade da doença reumática) e alterações neurológicas (doença de Parkinson e acidente vascular cerebral). A presença dessas alterações foi avaliada na anamnese inicial. Idosas que faltaram a algum encontro ou se recusaram a completar alguma avaliação também foram excluídas, assim como idosas com diagnóstico e/ ou suspeita de depressão, detectada por meio da Escala de Depressão Geriátrica de Yesavage (GDS-30) ${ }^{8-10}$.

Inicialmente, 70 mulheres idosas foram recrutadas. Destas, 25 foram excluídas: 9 por alterações neurológicas; 4 por escore GDS-30>11; 2 devido a alterações ortopédicas graves; 2 por se encontrarem em fase moderada e avançada da DA; 3 sem diagnóstico de demência; e 5 por faltarem a pelo menos uma das reuniões. As 45 idosas selecionadas foram divididas em dois grupos: saudáveis $(\mathrm{GIH}=27)$ e com $\mathrm{DA}(\mathrm{GIA}=18)$.

\section{Instrumentos}

A bateria constou de anamnese pré-elaborada contendo informações sociodemográficas, clínicas (comorbidades e número de medicamentos utilizados), prática de atividade física e histórico de quedas.

Para avaliação cognitiva, utilizaram-se os instrumentos Montreal Cognitive Assessment (MoCA) - uma ferramenta de rastreamento de curta duração para comprometimento cognitivo leve (CCL) ${ }^{11,12}$ - e Cambridge Cognitive Exam-Revised (CAMCOG-R), cujo objetivo é detectar estágios iniciais de síndromes de demência e prever o desenvolvimento da doença. Neste estudo, o CAMCOG- $\mathrm{R}$ foi usado especificamente para avaliar funções executivas e praxia ${ }^{13-15}$.

Para avaliação motora, foram utilizadas a Escala de Equilíbrio de Berg (EEB), que avalia o desempenho do equilíbrio funcional em 14 itens comuns da vida diária em uma ordem-padrão ${ }^{16}$, e o timed up and go test (TUGT), que avalia a mobilidade e o equilíbrio necessários para se levantar de uma cadeira, caminhar por três metros, voltar e sentar-se ${ }^{17}$.
Para avaliação funcional, utilizou-se a Avaliação do Estado Funcional Direto (DAFS-BR), que examina diretamente uma ampla gama de habilidades funcionais em pacientes idosos com e sem comprometimento cognitivo $^{18,19}$. Todas as escalas foram validadas para uso no Brasil.

\section{Procedimentos}

As avaliações foram divididas em dois encontros com duração de 1 hora e 30 minutos cada. No primeiro, foi feita a anamnese com dados sociodemográficos, clínicos e de queda, e os instrumentos MoCA e EEB foram aplicados. No segundo encontro, CAMCOG-R, TUGT e DAFS-BR foram aplicados. Os testes foram realizados por um avaliador treinado, a fim de minimizar possíveis desvios. A coleta das informações e a aplicação das escalas ocorreram entre os meses de fevereiro e junho de 2017.

No caso de idosas com DA, as informações clínicas foram coletadas previamente com familiares, que também confirmaram os dados em prontuário. Somente os idosos com DA diagnosticada por geriatra ou neurologista e com estadiamento de acordo com a Clinical Dementia Rating Scale (CDR-1: estágio leve/ estágio precoce da doença) foram incluídos no estudo. Os idosos e seus responsáveis legais (quando necessário) assinaram o termo de consentimento livre e esclarecido.

\section{Análise estatística}

Os resultados foram analisados no programa SPSS Statistics para Windows, versão 17.0 (Chicago: SPSS Inc., 2008), definindo o valor-p<0,05 para o nível de significância. As variáveis sociodemográficas e os escores dos testes passaram por análise descritiva.

Para analisar a distribuição dos dados, foram realizados os testes de Levene e Shapiro-Wilk. Para comparar os grupos e avaliar o tamanho do efeito, foi usado o teste Delta de Cliff. Para analisar a associação entre duas variáveis categóricas foram utilizados o teste do qui-quadrado de Pearson e o exato de Fisher.

O teste U de Mann-Whitney foi aplicado para avaliar a diferença entre os escores dos testes observacionais e o número de quedas entre os grupos. A análise de correlação de Spearman foi realizada entre os escores dos testes e o número de quedas. Dadas as características da amostra, realizou-se a regressão bootstrap $(n=10.000)$, estratificada pela variável grupo e com correção de viés. 


\section{Descrição da amostra}

Considerando o erro amostral de 5\%, o tamanho da amostra apontou um mínimo de 10 idosas em cada grupo. Trinta e cinco mulheres idosas foram avaliadas, 27 do GIH e 18 do GIA, na fase leve da doença (CDR 1). As principais características sociodemográficas, psicomotoras, cognitivas e funcionais estão descritas na Tabela 1. Não foi observada diferença significativamente estatística entre os grupos com relação a renda $(\mathrm{p}=0,18)$, porém os itens escolaridade e idade apresentaram alterações significativas entre os grupos ( $p=-0,51$ e $p=0,33$ respectivamente). Com relação a escolaridade, apesar da diferença apontada, o cálculo do delta de Cliff apontou uma pontuação de 0,33 (que representa um efeito pequeno).Já com relação a idade, a análise da covariância (Ancova) apontou os seguintes resultados: não há efeito da idade sobre o resultado da escala motora TUG [F $(1,42)=2,185 ; \mathrm{p}=, 147]$; idade sobre resultado da escala cognitiva $\mathrm{MoCA}[\mathrm{F}(1,42)=, 362$; $\mathrm{p}=0,551]$; idade sobre o resultado da escala motora de Berg $[\mathrm{F}(1,42)=3,185 ; \mathrm{p}=0,083]$; idade sobre o resultado da escala cognitiva CAMCOG-R [F $(1,42)=, 081 ; \mathrm{p}=, 778]$ e idade sobre o resultado da escala funcional DAFS-BR $[\mathrm{F}(1,42)=, 115 ; \mathrm{p}=, 737]$.

Informações sobre aspectos de saúde foram coletadas e analisadas. Encontraram-se altas porcentagens de hipertensão arterial sistêmica (16,4\% em GIH e 16,3\% em GIA, $p=0,01)$, dislipidemia (16,4\% em GIH e 16,3\% em GIA, $p=0,01)$, alterações ortopédicas $(26,9 \%$ em GIH e 8,7\% em GIA, $p=0,1)$, osteoporose $(10,4 \%$ em $\mathrm{GIH}$ e $13 \%$ em GIA, $\mathrm{p}=0,01)$ e alterações visuais $(9 \%$ em GIH e $13 \%$ em GIA, $p=0,00$ ).

Tabela 1. Características sociodemográficas, clínicas e escores da escala (cognitiva, motora e funcional) dos grupos avaliados expressos em média e desvio-padrão

\begin{tabular}{|c|c|c|c|c|}
\hline $\begin{array}{l}\text { Caracterização da amostra } \\
\text { e escore das escalas }\end{array}$ & GIH & GIA & p & Delta de Cliff \\
\hline Idade (anos) & $79,4 \pm 5,2$ & $84,1 \pm 5,1$ & 0,00 & $-0,51$ \\
\hline Escolaridade (ano) & $11,0 \pm 3,4$ & $9,4 \pm 2,3$ & 0,04 & 0,33 \\
\hline Comorbidades (número) & $2,4 \pm 1,4$ & $5,1 \pm 1,9$ & 0,00 & $-0,72$ \\
\hline Medicações (número) & $1,9 \pm 0,9$ & $3,6 \pm 1,0$ & 0,00 & $-0,78$ \\
\hline Atividade Física (número) & $2,78 \pm 1,0$ & $1,89 \pm 0,6$ & 0,00 & 0,50 \\
\hline Quedas (número) & $0,2 \pm 0,4$ & $0,7 \pm 0,7$ & 0,02 & $-0,33$ \\
\hline MoCA FE & $3,6 \pm 1,2$ & $1,5 \pm 1,1$ & 0,00 & 0,74 \\
\hline Total MoCA & $23,7 \pm 3,2$ & $11,3 \pm 3,5$ & 0,00 & 0,99 \\
\hline TUGT & $11,8 \pm 2,7$ & $22,6 \pm 6,5$ & 0,00 & $-0,97$ \\
\hline Total EEB & $50,1 \pm 4,2$ & $36,2 \pm 8,2$ & 0,00 & 0,92 \\
\hline CAMCOG-R Praxia & $10,2 \pm 1,8$ & $6,9 \pm 2,6$ & 0,00 & 0,69 \\
\hline CAMCOG-R FE & $13,8 \pm 3,3$ & $5,2 \pm 1,8$ & 0,00 & 0,98 \\
\hline Total CAMCOG-R & $92,1 \pm 9,6$ & $50,3 \pm 11,9$ & 0,00 & 1,00 \\
\hline DAFS-BR Orientação & $16,0 \pm 0,0$ & $8,7 \pm 3,7$ & 0,00 & 0,94 \\
\hline DAFS-BR Comunicação & $14,1 \pm 1,1$ & $8,5 \pm 2,5$ & 0,00 & 0,94 \\
\hline DAFS-BR Dinheiro & $24,5 \pm 4,3$ & $10,3 \pm 3,7$ & 0,00 & 0,98 \\
\hline DAFS-BR Compras & $15,3 \pm 3,8$ & $3,0 \pm 2,4$ & 0,00 & 0,93 \\
\hline DAFS-BR Vestir/ Higiene & $13,0 \pm 0,0$ & $11,6 \pm 2,3$ & 0,00 & 0,61 \\
\hline DAFS-BR Alimentação & $10,0 \pm 0,0$ & $9,2 \pm 1,2$ & 0,00 & 0,33 \\
\hline Total DAFS-BR & $93,0 \pm 7,8$ & $51,6 \pm 11,1$ & 0,00 & 0,99 \\
\hline
\end{tabular}

*GIH: Grupo de Idosos Saudáveis. GIA: Grupo de idosos com doença de Alzheimer. MOCA FE: Funções executivas dos itens da Montreal Cognitive Assessment; TUGT: timed up and go test; EEB: Escala de Equilibrio de Berg; CAMCOG PRAXIA: item praxia do Cambridge Cognitive Exam-Revised; CAMCOG FE: item funções executivas do Cambridge Cognitive Exam-Revised; DAFS-BR: Avaliação Direta do Estado Funcional - Versão Brasileira. $p<0,05$. Teste U de Mann-Whitney e delta de Cliff.

\section{RESULTADOS}

\section{Padrões de perda psicomotora, cognitiva e funcional}

Os valores de referência dos testes cognitivos, motores e funcionais foram comparados com as médias obtidas no GIH e GIA. Depois, os itens foram classificados da seguinte forma: da maior para a menor diferença percentual entre a referência e os valores obtidos nos grupos. A Figura $1 \mathrm{~A}$ ilustra padrões de perda cognitiva. No GIH, as funções cognitivas com maior diferença percentual entre os valores obtidos e os valores de referência foram: praxia, funções executivas e abstração, enquanto no GIA foram: abstração, memória e funções executivas com Delta de Cliff=-1. 
A Figura 1B ilustra os padrões de perda motora. Em ambos os grupos, GIH e GIA, as duas habilidades motoras com maior diferença percentual entre os valores obtidos e os valores de referência foram: suporte unipodal e tandem. A terceira habilidade no GIH foi inclinar-se para frente, enquanto no GIA foi girar 360 graus com o efeito Delta de Cliff $=-1$.
A Figura 1C ilustra os padrões de perda funcional. No GIH, as funções com maior diferença percentual entre os valores obtidos e os de referência foram calcular o saldo da conta, dar troco correto e memória para seis itens de uma lista; enquanto no GIA foram usar o telefone, dar troco correto e memória para seis itens de supermercado com Delta de Cliff $=-1$.

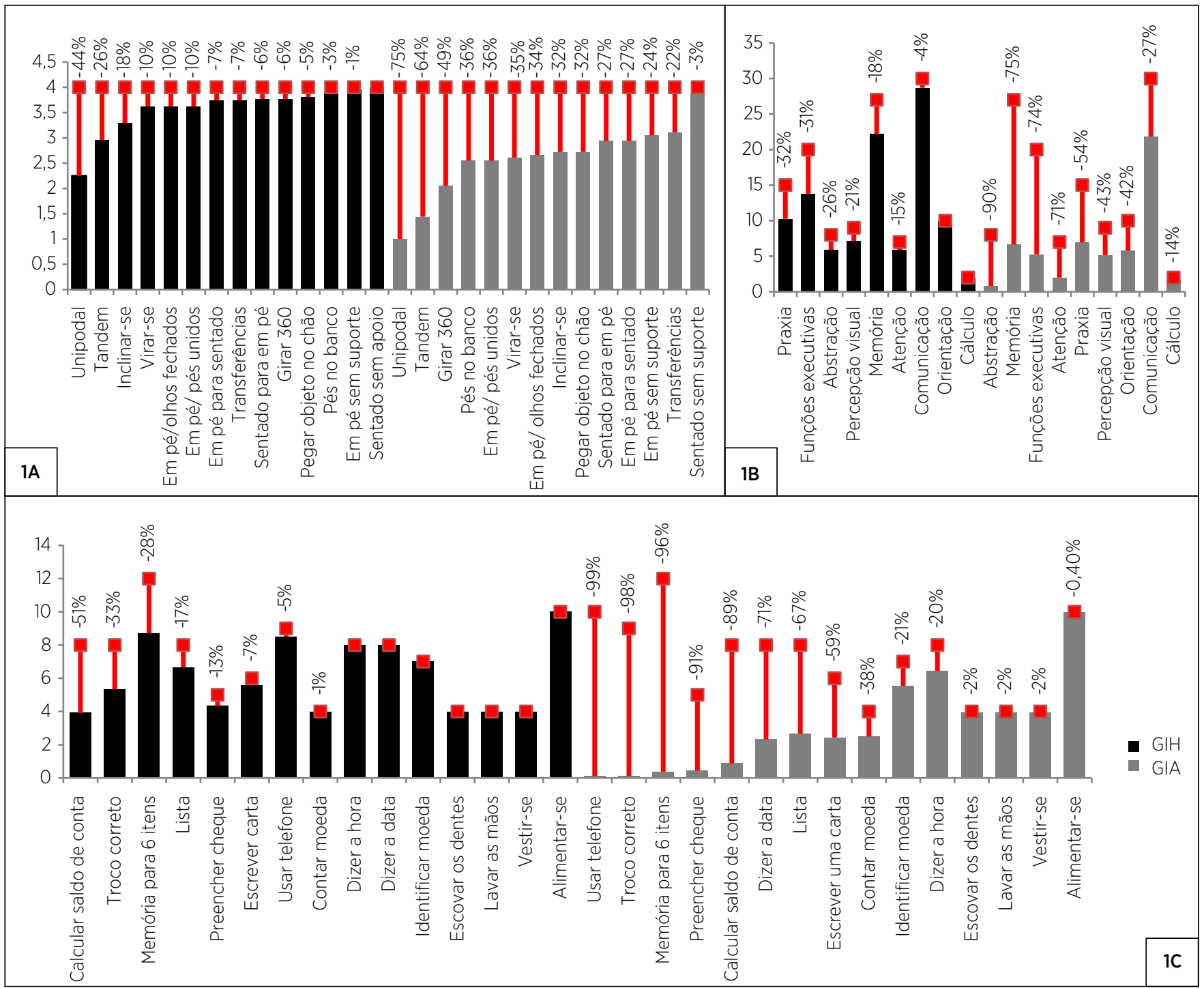

Figura 1. Gráfico comparativo com diferença entre os valores esperados e as médias obtidas nos aspectos motores, cognitivos e funcionais. 1A: padrões de perdas motoras; 1B: padrões de perdas cognitivas; e 1C: padrões de perdas funcionais. A unidade de medida adotada no gráfico é igual aos pontos. As colunas representam as médias obtidas pelos grupos e as linhas vermelhas representam a diferença para os valores esperados. "GIH: grupo de idosas hígidas; GIA: grupo de idosas com Doença de Alzheimer.

\section{Correlação e dependência entre variáveis cognitivas, motoras e funcionais}

Para detectar possíveis correlações entre as escalas cognitivas, motoras e funcionais, foi realizado o teste de correlação de Spearman. O item praxia $(\rho=-0,56$; p-valor $<0,00)$ bem como o item funções executivas $(\rho=-0,80 ; p$-valor $<0,00)$ do CAMCOG-R esteve altamente correlacionado com TUGT (velocidade da marcha). O mesmo ocorreu com Berg (equilíbrio) - praxia $(\rho=-0,59 ; p$-valor $<0,00)$ e funções executivas $(\rho=-0,76$; $\mathrm{p}$-valor $<0,00)$. Isso sugere uma provável relação entre 
essas funções cognitivas e o risco de cair. Também foi encontrada forte correlação entre os itens da escala de funcionalidade DAFS-BR com as escalas cognitivas MoCA e CAMCOG-R, principalmente os itens praxia $(\rho=0,76$; $p$-valor $<0,00)$ e funções executivas $(\rho=0,88$; $p$-valor $<0,00)$.

Para avaliar a relação de dependência das variáveis escolhidas nos dois grupos, foram construídos dois modelos: (1) BERG TOTAL = GRUPO + CAMCOG-R TOTAL e (2) DAFS-BR TOTAL = GRUPO + CAMCOG-R TOTAL. Os ajustes para os modelos foram excelentes, com: (a) $\mathrm{R}^{2}$ do modelo (1)=0,81 e $\mathrm{R}^{2}$ do modelo (2)=0,96; (b) Durbin-Watson próximo de 2 em ambos os modelos, indicando que a heterogeneidade e a autocorrelação não foram problemas; (c) medida $\mathrm{VIF}<10$ em ambos os modelos, indicando que a multicolinearidade não foi um problema; e, por fim, (d) ausência de outliers.

$\mathrm{Na}$ avaliação da relação de dependência entre as escalas de Berg e CAMCOG-R, observou-se significância estatística: a cada ponto aumentado no escore total de CAMCOG-R ocorre aumento da pontuação de Berg total em 0,27 ( $p$-valor<0,01). Não houve diferença entre os grupos, ou seja, a relação de dependência entre cognição, equilíbrio e risco de quedas foi semelhante tanto em idosas hígidas quanto em idosas com DA.

$\mathrm{Na}$ avaliação da relação de dependência entre as escalas DAFS-BR e CAMCOG-R, observou-se que o aumento de um ponto no total do CAMCOG-R implicou em aumento de 0,69 (p-valor<0,01) no DAFS-BR total. Houve diferença estatística entre os grupos. No GIH, o aumento de um ponto no CAMCOG-R total resultou em aumento de 12,49 pontos no DAFS-BR.

\section{DISCUSSÃO}

Seguindo a lógica proposta pela TR, o desenvolvimento torna-se (des)envolvimento, a evolução torna-se involução, e a organização vertical ascendente torna-se desorganização vertical descendente $\mathrm{f}^{6,7,20,21}$. O objetivo deste estudo foi avaliar e descrever aspectos psicomotores, cognitivos e funcionais e a relação de dependência entre essas variáveis em idosas saudáveis e diagnosticadas com DA provável na fase inicial. As hipóteses testadas foram: as primeiras perdas (cognitivas, motoras e funcionais) na DA seriam as das funções mais complexas; essas perdas obedeceriam à ordem inversa do desenvolvimento neuropsicomotor proposto por Piaget; e existiria correlação e relação de dependência entre cognição e funcionalidade e cognição e aspectos motores.
Do ponto de vista da funcionalidade, avaliada pela escala DAFS-BR, as atividades instrumentais (calcular saldo da conta, memorizar e selecionar itens de uma lista de compras, usar o telefone, preencher cheque) foram aquelas que apresentaram menores escores. A diferença, além da intensidade das perdas, reside no fato de que no GIA as atividades mais envolvidas foram aquelas que envolviam diretamente memória, funções executivas e praxia (memória para itens, utilizando telefone). $\mathrm{Na}$ análise estatística, houve relação de dependência entre funcionalidade e cognição, sendo que o aumento de um ponto no CAMCOG-R total implicou um aumento de 0,69 ( $\mathrm{p}$-valor<0,01) no DAFS-BR total. Houve diferença estatística entre GIH e GIA. Em GIH, o aumento de um ponto no total CAMCOG-R resultou em um aumento de 12,49 pontos no DAFS-BR. Isso indica uma provável relação entre a capacidade cognitiva e a capacidade de realizar atividades instrumentais (mais complexas). Deve-se notar que esse resultado era esperado, uma vez que o diagnóstico da depende do declínio funcional progressivo associado ao comprometimento cognitivo. E o DAFS-BR é uma escala que mede a funcionalidade.

Reisberg et al. ${ }^{6}$ sugeriram essa mesma inversão de níveis funcionais a partir dos dados obtidos por outro instrumento: Functional Assessment Staging Test (FAST). As perdas funcionais observadas ocorreram na seguinte ordem: perda de trabalho, necessidade de assistência em atividades complexas, auxílio na escolha de roupas, assistência com banho e alimentação ${ }^{6,20}$. Um estudo transversal realizado no Sul do Brasil encontrou resultados muito semelhantes aos obtidos nesse estudo: as atividades instrumentais mais complexas foram afetadas desde a fase inicial $\mathrm{da}-2 \%$ dos idosos avaliados apresentaram dependência total para atividades instrumentais da vida diária (AIVD)já na fase leve da doença. $\mathrm{Na}$ fase grave, essa porcentagem subiu para $29,3 \%{ }^{22}$.

Estudos sugerem que a disfunção executiva é o fator central envolvido em problemas nas atividades da vida diária (AVD). Essa disfunção pode estar associada à atrofia cortical observada nas fases graves da, que acontece em áreas motoras do córtex frontal e em áreas de integração sensório-motora, interferindo diretamente na função ${ }^{23}$. Perdas na praxia fina (agilidade e precisão), por exemplo, prejudicam diretamente a funcionalidade e o desempenho nas tarefas cotidianas ${ }^{24}$. A circuitaria da praxia envolve o sistema cortical do hemisfério dominante, que por sua vez ativa o córtex pré-motor do hemisfério esquerdo. Quando este é ativado, um sinal é enviado para o hemisfério direito, onde o corpo caloso transmite o sinal até o 
córtex motor do hemisfério direito, possibilitando o ato práxico $^{25}$. Apesar da associação comum com demências vasculares, um estudo de Yoshii et al. ${ }^{26}$ encontrou que o comprometimento da praxia ocorreu tanto em nível cortical quanto em subcortical ${ }^{26}$.

Com relação à cognição, esta pesquisa utilizou dois instrumentos de avaliação: MoCA e CAMCOG-R, considerando a forte correlação entre as escalas $(\rho=0,920$, valor-p<0,01) e o fato de que a escala CAMCOG-R apresenta um maior número de funções cognitivas avaliadas (especificamente funções executivas e praxia) para análises de correlação subsequentes. Em uma análise de funções cognitivas com escores mais baixos, o GIH apresentou alterações (não indicando comprometimento) em: praxia, funções executivas, abstração e percepção visual. A GIA apresentou perdas significativas em abstração, memória, funções executivas e atenção. Tal informação está de acordo com os declínios cognitivos descritos na $\mathrm{DA}^{27,28}$. A memória recente seria inicialmente afetada, com consequente desorientação espacial e dificuldade em aprender, codificar, armazenar, recuperar e sequenciar ações ${ }^{29,30}$. O prejuízo das funções executivas se justificaria pelo envolvimento de regiões frontais nos estágios iniciais da $\mathrm{DA}^{31,32}$.

O comprometimento das funções cognitivas também pode influenciar a parte motora, o equilíbrio e o risco de queda. Avaliando e comparando as médias obtidas entre os grupos no TUGT, observou-se que a velocidade média de caminhada do GIA foi muito inferior à encontrada no GIH. Isso significa que os idosos com DA realizaram o teste por mais tempo (desacelerando), o que sugere maior risco de queda. Um estudo anterior encontrou resultados semelhantes aplicando a mesma escala em idosos com DA na fase leve ${ }^{33}$. Pacientes idosos com DA inicial tendem a aumentar a modulação do padrão locomotor em relação ao estágio pré-demencial, o que torna a marcha mais cautelosa e, consequentemente, reduz sua velocidade ${ }^{34}$. A redução da velocidade da marcha também foi corroborada por estudo longitudinal realizado em 2017, que observou como idosos com CCL e DA em fase leve apresentavam menor velocidade de marcha quando comparados a idosos saudáveis, ainda que essa redução não tenha sido capaz de prever quedas em nenhum dos grupos avaliados ${ }^{35}$.

$\mathrm{Na}$ avaliação do equilíbrio pelo $\mathrm{EEB}$, os escores médios finais obtidos nos grupos diferiram significativamente. $\mathrm{O}$ GIA apresentou escores inferiores aos do GIH, indicando maiores alterações no equilíbrio e maior risco de queda. Outros estudos corroboram essa pesquisa, encontrando resultados semelhantes ${ }^{36}$.
Os itens de equilíbrio mais comprometidos no GIA foram os que exigiram maior controle motor (postura complexa) ou planejamento motor (sequência de movimentos), enquanto posturas mais baixas (sedestação) não apresentaram alterações. Essa observação pode sugerir um padrão de perda motora e referir-se ao proposto pela TR: o comprometimento de posturas mais baixas só é percebido na fase grave da doença, quando os idosos costumam assumir posturas flexoras associadas a reflexos primitivos.

As duas escalas escolhidas para avaliar o risco de quedas se mostraram altamente correlacionadas $(\rho=-0,89)$. Para análise de regressão linear, optou-se pela utilização do $\mathrm{EEB}$, que divide o equilíbrio em estágios motores com diferentes níveis de dificuldade.

Foi encontrada correlação entre o escore CAMCOG-R e o número de quedas nos dois grupos $(\rho=-0,32$, $\mathrm{p}$-valor=0,03), ou seja, quanto menor o escore total, maior o número de quedas observado. Estudos anteriores indicam que o declínio cognitivo é uma variável que pode influenciar diretamente o risco de quedas em idosos ${ }^{37,38}$. Em 2017, Lee et al..$^{37}$ investigaram a associação entre os volumes subcorticais de substância cinzenta e instabilidade postural na DA, observando que todas as estruturas cerebrais, exceto o globo pálido, foram menores em comparação com o grupo-controle. Os idosos com DA apresentaram desempenho ruim nos testes de postura unilateral e organização sensorial na plataforma de equilíbrio. Esse mesmo grupo apresentou aumento da frequência de quedas ${ }^{37}$.

Em estágios leves da DA, o risco de quedas é semelhante ao risco de quedas em idosos saudáveis ${ }^{36}$. Ansai et al..$^{39}$, em um estudo transversal, também não encontraram diferenças significativas na marcha e no risco de quedas nos grupos de idosos sem comprometimento cognitivo, com CCL e com DA leve, porém observaram diferença significativa na dupla tarefa no grupo DA leve. Esse achado aponta para a possível interferência dos componentes cognitivos nos episódios de quedas, visto que a dupla tarefa envolve funções executivas ${ }^{39}$. No entanto, à medida que a doença progride e compromete as áreas corticais, componentes motores mais simples, como sentar e levantar (na fase moderada da), também são comprometidos, o que aumenta ainda mais o risco de quedas ${ }^{40}$. Deve-se levar em consideração que nas fases moderada e grave da doença o uso de medicamentos antidepressivos e antipsicóticos pode predispor a um número ainda maior de quedas ${ }^{41}$. 
Ambas as funções executivas e praxia foram altamente correlacionadas com o risco de quedas avaliado pelo TUGT e pela EEB. Observou-se uma relação de dependência entre o CAMCOG-R e a EEB: onde em cada ponto houve aumento no escore total do CAMCOG-R, houve aumento no escore da EEB. No entanto, não houve diferença entre os grupos. A relação entre dependência e cognição, equilíbrio e risco de quedas foi semelhante em mulheres saudáveis e idosas com DA. Um estudo anterior encontrou uma correlação negativa entre a função cognitiva do mini exame do estado mental (MEEM) e a agilidade do equilíbrio (AGILEQ) em pacientes idosos com DA, indicando também que o desempenho em testes motores poderia estar diretamente associado ao nível de funções cognitivas do paciente ${ }^{42}$. Outro estudo constatou que prejuízos da função cognitiva poderiam aumentar proporcionalmente a disfunção da marcha e, consequentemente, aumentar o risco de quedas ${ }^{43}$.

Entender a ordem de perda de funções cognitivas, funcionais e motoras, bem como a relação entre elas, pode auxiliar no desenvolvimento de terapias mais assertivas, que possam ser aplicadas no início da. A perda de funções cognitivas complexas, além da TR, pode ser explicada pela neurodegeneração global e consequente perda de conectividade entre redes cerebrais. Pensando nisso, seria interessante que estudos futuros realizassem monitoramento longitudinal e monitoramento de resultados por meio de testes de neuroimagem.

$\mathrm{O}$ estudo apresentou algumas limitações. Por ser transversal, não foi possível comparar os idosos ao longo do tempo. Sugere-se que estudos longitudinais sejam realizados para melhor compreender os padrões de perda na DA e validar a TR.

Neste estudo, o grupo GIA apresentou média de idade e comorbidades significativamente maiores que o GIH, o que pode sugerir um maior comprometimento funcional $\mathrm{e}$ motor. É importante ressaltar a dificuldade no diagnóstico precoce de pacientes com DA e a dificuldade de acesso a esses pacientes.

\section{CONCLUSÃO}

Foi observada relação de dependência entre funcionalidade e cognição e equilíbrio e cognição, bem como correlação entre risco de queda e desempenho cognitivo. Além disso, foi possível sugerir uma ordem nas perdas funcionais, cognitivas e motoras que vai ao encontro da teoria proposta, segundo a qual nas fases iniciais da ocorrem perdas de habilidades funcionais instrumentais, funções cognitivas complexas (memória, funções executivas e praxia), redução da velocidade da marcha e redução do equilíbrio em posturas que exigem mais planejamento e controle motor, resultando em maior chance de queda. Houve alta correlação entre os itens praxia e funções executivas do CAMCOG-R com TUGT e EEB e o risco de quedas.

\section{AGRADECIMENTOS}

Agradecemos o apoio do Programa de Pós-Graduação em Neurociências e Cognição da Universidade Federal do ABC (UFABC), da Fundação Nossa Senhora Auxiliadora do Ipiranga (Funsai), da Associação de Familiares e Amigos do Idoso (Afai) e da Associação de Pais e Amigos dos Excepcionais de São Paulo (Apae).

\section{REFERÊNCIAS}

1. Alzheimer's Disease International. World Alzheimer Report 2015: the global impact of dementia: an analysis of prevalence, incidence, cost and trends. London: Alzheimer's Disease International; 2015.

2. Delaby C, Gabelle A, Blum D, Schraen-Maschke S, Moulinier A, Boulanghien J, et al. Central nervous system and peripheral inflammatory processes in Alzheimer's disease: biomarker profiling approach. Front Neurol. 2015;6:181. doi: 10.3389/ fneur.2015.00181

3. von Bernhardi R, Cornejo F, Parada GE, Eugenín J. Role of TGF $\beta$ signaling in the pathogenesis of Alzheimer's disease. Front Cell Neurosci. 2015;9:426. doi: 10.3389/fncel.2015.00426

4. Ardilla A. Normal aging increases cognitive heterogeneity: analysis of dispersion in WAIS-III scores across age. Arch Clin Neuropsychol. 2007;22(8):1003-11. doi: 10.1016/j. acn.2007.08.004

5. Farfel JM. Fatores relacionados à senescência e à senilidade cerebral em indivíduos muito idosos: um estudo de correlação clinicopatológica [thesis]. São Paulo: Universidade de São Paulo; 2008. 157 p.

6. Reisberg B, Franssen EH, Hasan SM, Monteiro I, Boksay I, Souren LEM, et al. Retrogenesis: clinical, physiologic and pathologic mechanisms in brain aging, Alzheimer's and other dementing processes. Eur Arch Psychiatric Clin Neurosci. 1999;249(3):28-36. doi: 10.1007/pl00014170

7. Reisberg B, Franssen EH, Souren LEM, Kenowsky S, Jamil IA, Anwar S, Auer S. Alzheimer's disease. In: Flanagan SR, Zaretsky H, Moroz A, editors. Medical aspects of disability: a handbook for the rehabilitation professional. 4th ed. New York: Springer Publishing Company; 2011. p. 25-65.

8. Yesavage JA, Brink TL, Rose TL, Lum O, Huang V, Adey M, Leirer VO. Development and validation of a geriatric depression 
screening scale: a preliminary report. J Psychiatr Res. 19821983;17(1):37-49. doi: 10.1016/0022-3956(82)90033-4

9. Farrel C. Poststroke depression in elderly patients. Dimens Crit Care Nurs. 2004;23(6):264-9. doi: 10.1097/00003465-200411000-00007

10. Roman MW, Callen BL. Screening instruments for older adult depressive disorders: updating the evidence-based toolbox. Issues Ment Health Nurs. 2009;29(9):924-41. doi: 10.1080/01612840802274578

11. Nasredinne ZS, Phillips NA, Bédirian V, Charbonneau S, Whitehead V, Collin I, et al. The Montreal Cognitive Assessment, MoCA: a brief screening tool for mild cognitive impairment. J Am Geriatr Soc. 2005;53(4):695-9. doi: 10.1111/j.1532-5415.2005.53221.x

12. Smith T, Gildeh N, Holmes C. The Montreal Cognitive Assessment: validity and utility in a memory clinic setting. Can J Psychiatry. 2007:52(5):329-32. doi: 10.1111/j.1532-5415.2005.53221.x

13. Paradela, EMP. Adaptação transcultural para o português do Teste Cognitivo Cambridge Revisado e desempenho do teste em idosos ambulatoriais [thesis]. Rio de Janeiro: Universidade do Estado do Rio de Janeiro; 2007. 180 p.

14. Pereiro AX, Ramos-Lema S, Juncos-Rabadán O, Facal D, Lojo-Seoane C. Normative scores of the Cambridge Cognitive Examination-Revised in healthy Spanish population. Psicothema. 2015;27(1):32-9. doi: 10.7334/psicothema2014.169

15. Moreira IFH, Bezerra AB, Sudo FK, Alves GS, Ericeira-Valente L, Tiel $C$ et al. CAMCOG: valores das subescalas em idosos normais com níveis diferentes de escolaridade: aspectos preliminares. Rev Bras Neurol. 2013;49(1):32-6.

16. Miyamoto ST, Lombardi I Jr, Berg KO, Ramos LR, Natour J. Brazilian version of the Berg balance scale. Braz J Med Biol Res. 2004;37(9):1411-21. doi: 10.1590/S0100-879X2004000900017

17. Hofheinz $M$, Schusterschitz C. Dual task interference in estimating the risk of falls and measuring change: a comparative, psychometric study of four measurements. Clin Rehabil. 2010;24(9):831-42. doi: 10.1177/0269215510367993

18. Loewestein DA, Amigo E, Duara R, Guterman A, Hurwitz D, Berokowitz $\mathrm{N}$ et al. A new scale for the assessment of functional status in Alzheimer's disease and related disorders. J Gerontol. 1989;44(4):114-21. doi: 10.1093/geronj/44.4.p114

19. Pereira FS. Executive function and functional status in normal elderly, mild cognitive impairment and Alzheimer disease [dissertation]. São Paulo: Universidade de São Paulo; 2009.

20. Reisberg B, Franssen H, Souren LEM, Auer SR, Akram I, Kenowsky S. Evidence and mechanisms of retrogenesis in Alzheimer's and other dementias: management and treatment import. Am J Alzheimers Dis Other Demen. 2002;17(4):202-12. doi: 10.1177/153331750201700411

21. Rogers H, Lasprilla JCA. Retrogenesis theory in Alzheimer's disease: evidence and clinical implications. An Psicol. 2006;22(2):260-66.

22. Haskel MVL, Bonini JS, Santos SC, Silva WCFN, Bueno CFO, Bortolanza MCZ, Daniel CR. Funcionalidade na Doença de Alzheimer leve, moderada e grave: um estudo transversal. Acta Fisiatr. 2017;24(2):82-85

23. Pini L, Pievania N, Bocchetta M, Altomare D, Bosco P, Cavedo E, et al. Brain atrophy in Alzheimer's Disease and aging. Ageing Res Rev. 2016;30:25-48. doi: 10.1016/j.arr.2016.01.002
24. Andreatto CAA. Percepção de tempo e outras funções cognitivas, funcionalidade motora e o nível de atividade física em idosos com Doença de Alzheimer no estágio leve [dissertation]. Rio Claro: Universidade Estadual Paulista Júlio de Mesquita Filho, 2013. $126 \mathrm{f}$.

25. Moreira L, de Paula JJ. Praxia e visioconstrução. In: MalloyDiniz L, Fuentes D, Mattos P, Abreu N, editors. Avaliação neuropsicológica. 2nd ed. Porto Alegre: Artmed; 2018. p. 106-11.

26. Yoshii F, Kawaguchi C, Kohara S, Shimizu M, Onaka H, Ryo M, Takahashi W. Characteristic deterioration of ADAS-Jcog subscale scores and correlations with regional cerebral blood flow reductions in Alzheimer's disease. Neurol Sci. 2018;39(5):909-18. doi: 10.1007/s10072-018-3277-6

27. Arango-Lasprilla JC. Alteraciones neuropsicológicas en la enfermedad de Alzheimer. In: Londoño PG, editor. Memoria y demencias. Neiva: Universidad Surcolombiana; 2004. p. 77-86.

28. Mckhann GM, Knopman DS, Chertkow H, Hyman BT, Jack CR Jr, Kawas $\mathrm{CH}$, et al. The diagnosis of dementia due to Alzheimer's disease: recommendations from the National Institute on AgingAlzheimer's Association workgroups on diagnostic guidelines for Alzheimer's disease. Alzheimers Dement. 2011;7(3):263-69. doi: 10.1016/j.jalz.2011.03.005

29. Borges S, Aprahamian I, Radanovic M, Forlenza OV. Psicomotricidade e retrogênese: considerações sobre o envelhecimento e a doença de Alzheimer. Rev Psiquiatr Clín. 2010;37(3)131-7. doi: 10.1590/S0101-60832010000300007

30. Caramelli E, Zinger-Vakin T, Morad M, Merrick J. Can physical training have an effect on well-being in adults with mild intellectual disability? Mech Ageing Den. 2005;126(2):299304. doi: 10.1016/j.mad.2004.08.021

31. Yaari R, Corey-Bloom J. Alzheimer's disease. Semin Neurol. 2007;27(1):32-41. doi: 10.1055/s-2006-956753

32. Ávila R, Miotto EC. Funções executivas no envelhecimento normal e na doença de Alzheimer. J Bras Psiquiatr. 2003;52(1):53-63.

33. Pedroso R. Relação entre nível de atividade física, cognição, processamento da informação e funcionalidade motora de idosos no estágio leve da doença de Alzheimer [dissertarion]. Rio Claro: Universidade Estadual Paulista Júlio de Mesquita Filho, 2012. 149 p.

34. Scarmeas N, Albert M, Brandt D, Blacker G, Hadjigeorgiou A, Papadimitriou B et al. Motor signs predict poor outcomes in Alzheimer disease. Neurology. 2005;64(10):1696-703. doi: 10.1212/01.WNL.0000162054.15428.E9

35. Massé FAA. Velocidade da marcha como preditor de quedas em idosos com transtorno neurocognitivo e doença de Alzheimer [dissertation]. São Carlos: Universidade Federal de São Carlos, 2017. 72 p.

36. Kato-Narita EM, Nitrini R, Radanovic M. Assessment of balance in mild and moderate stages of Alzheimer's disease: implications on falls and functional capacity. Arq Neuro-Psiquiatr. 2011;69(2):202-7. doi: 10.1590/S0004-282X2011000200012

37. Lee YW, Lee HMD, Chung IS, Yi HA. Relationship between postural instability and subcortical volume loss in Alzheimer's disease. Medicine. 2017;96(25):1-7. doi: 10.1097/ MD.0000000000007286

38. Christofoletti G, Oliani MM, Gobbi LTB, Gobbi S, Stella F. Risco de queda em idosos com doença de Parkinson e demência 
de Alzheimer: um estudo transversal. Rev Bras Fisioterapia. 2006;10(4):429-33. doi: 10.1590/S1413-35552006000400011

39. Ansai JH, Andrade LP, Rossi PG, Takahashi ACM, Vale FAC, Rebelatto JR. Gait, dual task and history of falls in elderly with preserved cognition, mild cognitive impairment and mild Alzheimer's disease. Braz J Phys Ther. 2017;21(2):144-51. doi: 10.1016/j.bjpt.2017.03.010

40. Manckoundia P, Mourey F, Pfitzenmeyer P, Papaxanthis C. Comparison of motor strategies in sit-to-stand and back-tosit motions between healthy and Alzheimer's disease elderly subjects. Neuroscience. 2006;137(2):385-92. doi: 10.1016/j. neuroscience.2005.08.079

41. Wei YJ, Simoni-Wastila L, Lucas JA, Brandt N. Fall and fracture risk in nursing home residents with moderate-to-severe behavioral symptoms of Alzheimer's disease and related dementias initiating antidepressants or antipsychotics. J Gerontol A Biol Sci Med Sci. 2017;72(5),695-702. doi: 10.1093/gerona/ glw095

42. Hernandez SSS, Coelho FGM, Gobbi S, Stella F. Efeitos de um programa de atividade física nas funções cognitivas, equilíbrio e risco de quedas em idosos com demência de Alzheimer. Rev Bras Fisioter. 2010;14(1):68-74. doi: 10.1590/ S1413-35552010000100011

43. Gonçalves J, Ansai JH, Masse FAA, Vale FAC, Takahashi ACM, Andrade LP. Dual-task as a predictor of falls in older people with mild cognitive impairment and mild Alzheimer's disease: a prospective cohort stud. Braz J Phys Ther. 2018;22(5):417-423. doi: 10.1016/j.bjpt.2018.03.011 